Draft VERSiOn MARCH 8, 2021

Preprint typeset using $\mathrm{LAT}_{\mathrm{E}} \mathrm{X}$ style AASTeX6 v. 1.0

\title{
STUDY OF DARK-MATTER ADMIXED NEUTRON STARS USING THE EQUATION OF STATE FROM THE ROTATIONAL CURVES OF GALAXIES
}

\author{
Z. REZAEI \\ Physics Department and Biruni Observatory \\ College of Sciences, Shiraz University \\ Shiraz 71454, Iran
}

\begin{abstract}
In this work, we employ the dark matter equations of state (DMEOSs) obtained from the rotational curves of galaxies as well as the fermionic DMEOS with $m=1.0 \mathrm{GeV}$ to study the structure of darkmatter admixed neutron stars (DMANSs). Applying the equation of state in the Skyrme framework for the neutron matter (NM), we calculate the mass-radius relation for different DMANSs with various DMEOSs and central pressure of dark matter (DM) to NM ratios. Our results show that for some DMEOSs, the mass-radius relations are in agreement with new observations, e.g. EXO 1745-248, $4 \mathrm{U}$ 1608-52, and $4 \mathrm{U}$ 1820-30, which are inconsistent with the normal neutron stars. We conclude that both DMEOSs and central pressure ratios of DM to NM affect the slope of the mass-radius relation of DMANSs. This is because of the interaction between DM and NM, which leads to gravitationally or self-bound DMANSs. We study the radius of the NM sphere as well as the radius of the DM halo for different DMANSs. The results confirm that, in some cases, a NM sphere with a small radius is surrounded by a halo of DM with a larger radius. Our calculations verify that, due to the different degrees of DM domination in DMANSs, with a value of the visible radius of a star two possible DMANSs with different masses can be exist. The gravitational redshift is also calculated for DMANSs with different DMEOSs and central pressure ratios. The results explain that the existence of DM in a DMANS leads to higher values of gravitational redshift of the star.
\end{abstract}

Keywords: stars: neutron - dark matter - galaxies: halos - galaxies: kinematics and dynamics equation of state.

\section{INTRODUCTION}

A neutron star is a compact star which contains many neutrons. The theory of general relativity predicts the existence of a maximum mass above which the neutron star is unstable and collapses into a quark star or black hole. The maximum mass of a neutron star depends on the interaction between particles, and thus the equation of state (EOS) of the system. The stiffer the EOS, the larger the maximum mass of neutron star. In addition, the rotation of the neutron star leads to higher values of the maximum mass. Since the neutron star contains dense asymmetric nuclear matter, one should apply the EOS of strongly interacting asymmetric nuclear matter to investigate the bulk properties of the star. In addition, some authors have studied the effects of the existence of other particles, such as hyperons, meson condensates, and quarks, in the core of neutron stars on the maximum mass (Ozel 2006; Alford et al. 2007; Li et al. 2010).

From observation, radio pulsars show large masses of about $2 M_{\odot}$ (Ozel 2006; Demorest et al. 2010; Antoniadis et al. 2013). For example, according to (Ozel 2006), the mass of neutron star EXO 07482676 is measured to be $2.10 \pm 0.28 M_{\odot}$ and the radius equal to $13.8 \pm 1.8 \mathrm{~km}$. From theory, stiff EOSs like the neutron matter (NM) EOS in SLy230a and SLy230b models (Chabanat et al. 1997), can result in such large masses and mass-radius relations. Nevertheless, in some observations-such as EXO 1745-248 with mass $M=1.4 \pm 0.1 M_{\odot}$ and radius $R=11 \pm 1 \mathrm{~km}$ (Ozel \& Guver \& Psaltis 2009), the neutron star in $4 \mathrm{U} 1608-52$ with mass $M=1.74 \pm 0.14 M_{\odot}$ and radius $R=9.3 \pm 1.0 \mathrm{~km}$ (Guver et al. 2010A), and also the neutron star in the low-mass X-ray binary 4U 1820-30 with mass $M=1.58 \pm 0.06 M_{\odot}$ and radius $R=9.1 \pm 0.4 \mathrm{~km}$ (Guver et al. 2010B),- the results are different. It can be shown that soft EOSs are needed to lead these mass-radius relations from observation. Different equations of state for pure nuclear matter that have been obtained using different methods, such as the variational method with 
$A V_{18}$ potential plus the UIX potential or $A V_{18}$ potential plus a three-body UIX potential (Akmal \& Pandharipande 1997), and Dirac-Brueckner-Hartree-Fock models (Prakash et al. 1988; Engvik et al. 1994) cannot predict some observational data, such as 4U 1608-52 and 4U 1820-30 (Lattimer \& Prakash 2001, 2007). Therefore, this rules out the possibility of the existence of the pure nuclear matter in the neutron star. One explanation for these observations is that the existence of some particles, such as quarks, mesons, and hyperons, in the neutron star can lead to soft EOSs and the mass-radius relations in agreement with these observations (Weber 2005; Page \& Reddy 2006; Alford et al. 2007; Klahn et al. 2007).

In addition, another possibility that leads to these mass-radius relations is the existence of dark matter (DM) in the neutron star. Cosmological structure (Springel et al. 2005), gravitational lensing (Massey et al. 2007), and galactic rotational curve (Weber \& de Boer 2010) are some observations that could confirm the existence of DM. According to (Ade et al. 2014), the contributions of ordinary matter, DM, and dark energy in the universe are $4.9 \%$, $26.8 \%$, and $68.3 \%$, respectively. Supposing the existence of DM, it should be present in all astrophysical objects (Sandin \& Ciarcelluti 2009). The DM may be accreted to the neutron stars because of the large density of the neutron star matter (Bertone \& Fairbairn 2008; Perez-Garcia \& Silk 2012; Fuller \& Ott 2015). Therefore, to study models of DM, neutron stars are of much interest in astrophysics and astroparticle physics (Goldman \& Nussinov 1989; Bertone \& Fairbairn 2008; Kouvaris 2012). It has been shown that self-annihilating neutralino WIMP DM accreted onto neutron stars may lead to seeding compact objects with long-lived lumps of strange-quark matter (Perez-Garcia \& Silk \& Stone 2010). The heating caused by possible DM annihilation in neutron stars may be an observable (Lavallaz \& Fairbairn 2010) since the neutron star itself does not burn. In addition, it has been indicated that self-annihilation of DM in neutron stars can change their linear and angular momentum (Perez-Garcia \& Silk 2012), as well as the cooling properties of neutron stars (Kouvaris 2008). The critical phases of stellar evolution could be modified in the presence of relatively small amounts of DM (Sandin \& Ciarcelluti 2009).

Because of the possibility of the existence of DM in neutron stars, a model called the dark-matter admixed neutron star (DMANS) has been considered (Sandin \& Ciarcelluti 2009; Ciarcelluti \& Sandin 2011; Leung \& Chu \& Lin 2011, 2012; Xiang et al. 2014). The mechanisms for accumulating DM in a neutron star can result both from the stellar formation process and from subsequent accumulation by accretion of DM particles during the stellar lifetime (Ciarcelluti \& Sandin 2011). The DM inside the stars alters the structure and may lead to the collapse of a neutron star (Kouvaris 2012). The structure of these stars is still an open issue because of the unknown nature of DM. However, it is useful to study the structure of DMANSs using existing information on DM. The DMANS is a two-fluid system where the neutron star matter and DM interact only through gravitational force (Leung \& Chu \& Lin 2012). In order to study the structure of these stars, the Tolman-Oppenheimer-Volkoff (TOV) equation is separated into two different sets for the neutron star and dark components inside the star (Sandin \& Ciarcelluti 2009; Lavallaz \& Fairbairn 2010; Ciarcelluti \& Sandin 2011; Leung \& Chu \& Lin 2011, 2012).

The mass-radius relation of DMANSs which is affected by the DM, is one of the observational results that is possible to measure. The basic equation for DM to study the structure of DMANSs is the DM EOS (Sandin \& Ciarcelluti 2009; Ciarcelluti \& Sandin 2011; Leung \& Chu \& Lin 2011; Xiang et al. 2014). Applying a method that combines kinematic and gravitational lensing data to test the widely adopted assumption of pressureless DM, the DM EOS has been measured (Serra \& Dominguez Romero 2011). Moreover, by modeling galactic halos describing the DM as a nonzero pressure fluid and using observational data of the rotation curves of galaxies, a DM EOS has been obtained (Barranco \& Bernal \& Nunez 2015).

Some previous studies have been performed to investigate the structure of DMANSs (Sandin \& Ciarcelluti 2009; Ciarcelluti \& Sandin 2011; Leung \& Chu \& Lin 2011, 2012; Li \& Huang \& Xu 2012; Xiang et al. 2014). The general-relativistic hydrostatic equations have been generalized to spherical objects with multiple fluids that interact by gravity (Sandin \& Ciarcelluti 2009). Moreover, assuming that the microphysics is the same in the two sectors of the DMANSs, the effects of mirror DM on neutron star structure have been studied. It was concluded that the structure depends on the relative number of mirror baryons to ordinary baryons. Supposing that DM is made of some form of stable and long-living particles that can accumulate in the star, it has been shown that all mass-radius measurements can be explained with one nuclear matter EOS and a dark core of varying relative size (Ciarcelluti \& Sandin 2011). Consequently, observational data, which provide a test of the theory, will become a powerful tool for the determination of DM (Ciarcelluti \& Sandin 2011). Considering non-self-annihilating DM particles of mass $1.0 \mathrm{GeV}$ along with normal nuclear matter, and using a general relativistic two-fluid formalism, the properties of DMANSs have been studied (Leung \& Chu \& Lin 2011). It was found that a new class of compact stars consisting of a small normal matter core with radius of a few $\mathrm{km}$ within a $10 \mathrm{~km}$ DM halo can exist. Employing a general relativistic, two-fluid formalism to study the admixture of degenerate DM and normal nuclear matter shows that a new class of compact 


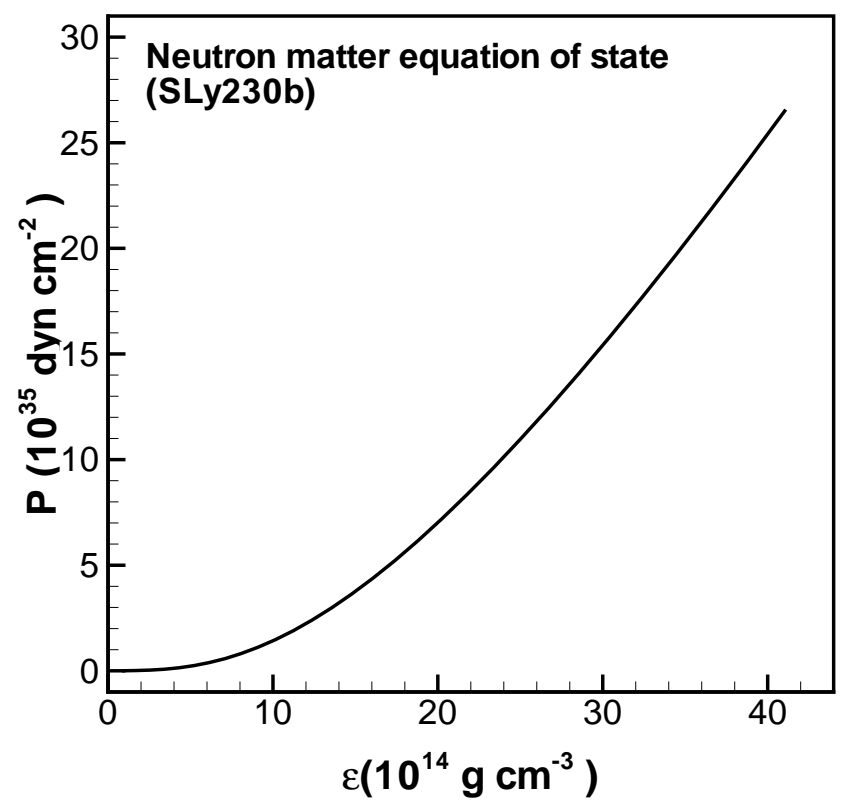

Figure 1. Neutron matter equation of state in the Skyrme framework, SLy230b (Chabanat et al. 1997).

stars that are dominated by DM can exist (Leung \& Chu \& Lin 2012). These stars have a small neutron star matter core, with radius of a few $\mathrm{km}$ embedded in a larger $10 \mathrm{~km}$ DM halo. In addition, these DMANSs have two classes of oscillation modes. The first class of modes reduces to the same set of modes for ordinary neutron stars without DM, and the second class of modes is due to DM. In a consistent DMANS model, considering DM particles to behave like fermions which interact with a certain repulsive interaction, it has been found that DM would soften the EOS more strongly than hyperons, reducing the maximum mass of the star ( $\mathrm{Li} \&$ Huang \& Xu 2012). However, with small mass particles, the maximum mass could be larger than $2 M_{\odot}$. The effects of fermionic DM on the properties of neutron stars using the two-fluid TOV formalism have been studied (Xiang et al. 2014). It has been shown that the mass of DM candidates, the amount of DM, and interactions among DM candidates affect the mass-radius relationship. In addition, the DM in a DMANS results in a spread of mass-radius relationships, and in some cases the DM distribution can surpass the NM distribution to form a DM halo. It has been confirmed that the DM admixture in neutron stars leads to the shrinkage of the NM surface, which results in the observation of a small radius. Moreover, the DM distribution can surpass the neutron star matter distribution and form a DM halo when the DM candidates have low mass or there is repulsion of DM. With a the large DM fraction in the DMANSs, the repulsive interaction among DM may result in stars with a mass of above $2 M_{\odot}$. In the present work, we investigate the properties of DMANSs using the Skyrme interaction in the neutron star matter and the DM EOS obtained from the rotational curves of galaxies in a general relativistic, two-fluid formalism.

\section{FORMALISM}

In this study, we employ the NM EOS that has been derived in the Skyrme framework (Chabanat et al. 1997). This equation is the result of the Skyrme effective force, SLy230b, with the improvement in the behavior with respect to the isospin degree of freedom (Chabanat et al. 1997). The EOS of NM in the SLy230b parametrization is shown in Figure 1. Recently, the DM EOS has been presented using the velocity profile of galaxies (Barranco \& Bernal \& Nunez 2015). In this study, we apply the pseudo isothermal model in which the DM density profile determines the velocity profile (Barranco \& Bernal \& Nunez 2015). The EOS for the pseudo-isothermal density profile is as follows (Barranco \& Bernal \& Nunez 2015),

$$
p(\rho)=\frac{8 p_{0}}{\pi^{2}-8}\left[\frac{\pi^{2}}{8}-\frac{\arctan \sqrt{\frac{\rho_{0}}{\rho}-1}}{\sqrt{\frac{\rho_{0}}{\rho}-1}}-\frac{1}{2}\left(\arctan \sqrt{\frac{\rho_{0}}{\rho}-1}\right)^{2}\right] .
$$



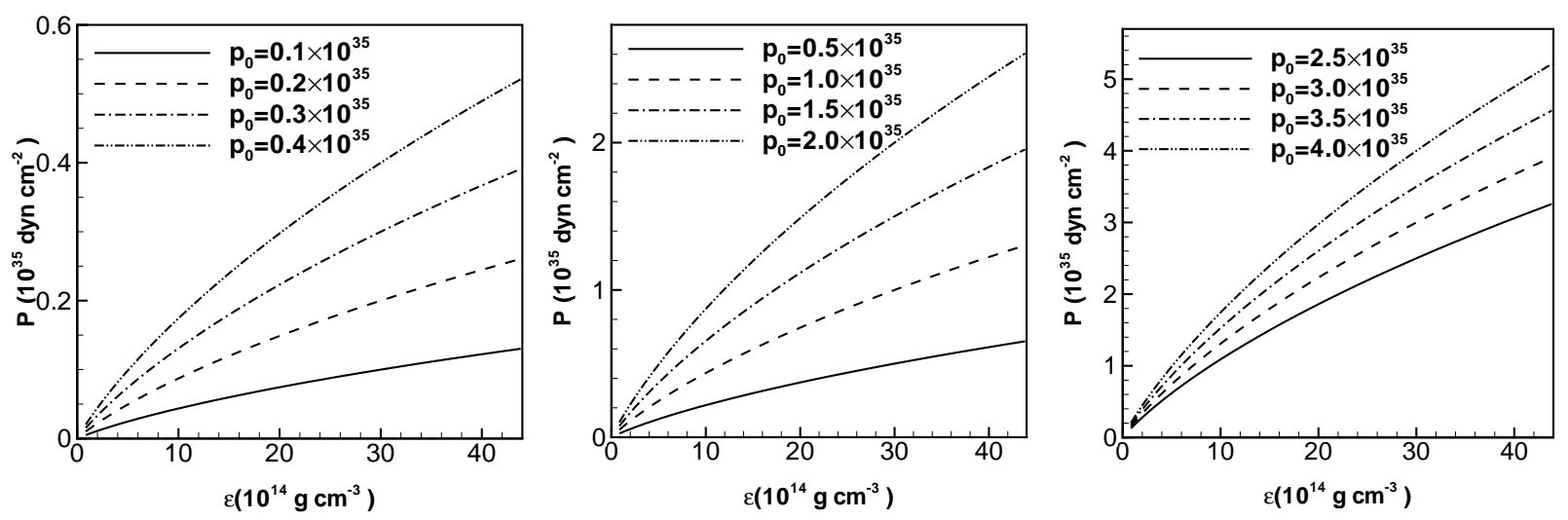

Figure 2. Dark matter equation of state from the velocity profile of galaxies with the value $\rho_{0}=0.3 \times 10^{16} \mathrm{~g} \mathrm{~cm}{ }^{-3}$ and different values of $p_{0}$ in $d y n \mathrm{~cm}^{-2}$ unit.

In the above equation, $\rho$ and $p$ are the density and pressure of DM, respectively. In addition, $\rho_{0}$ and $p_{0}$ are the only free parameters of the EOS, which for the DM in galaxies correspond to the central density and pressure, respectively (Barranco \& Bernal \& Nunez 2015). It has been found that this EOS has a functional dependence that is universal for all galaxies, and the mentioned free parameters are related to the evolution history of the galaxy. Using the EOS in Equation (1) and the rotational curve data, one can predict the central pressure and density of the galaxies. Here, we suppose that the DM in DMANSs also behaves according to this EOS. However, one should be careful about the value of the free parameters $\rho_{0}$ and $p_{0}$ in the EOS. A logical choice that is similar to the case of galaxies is that the values of $\rho_{0}$ and $p_{0}$ are of the order of the central density and pressure of neutron stars, respectively, i.e., $\sim 10^{15} \mathrm{~g} \mathrm{~cm}^{-3}$ for the density and $\sim 10^{36} \mathrm{dyn} \mathrm{cm}^{-2}$ for the pressure. We consider 12 EOSs with the value $\rho_{0}=0.3 \times 10^{16} \mathrm{~g} \mathrm{~cm}^{-3}$ and

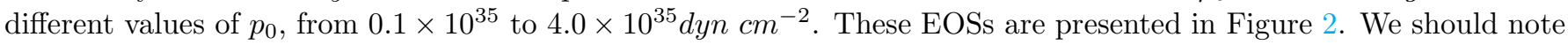
that we have used units in which $\mathrm{G}=\mathrm{c}=1$, and therefore the mass density and energy density of DM are equal, i.e., $\varepsilon=\rho$.

It is clear that the increase in the value of $p_{0}$ leads to more stiffness in the EOS. In addition, it is possible to consider DMANSs in which the DM sector is composed by free fermions with arbitrary masses (Narain et al. 2007). In this case the EOS at zero temperature, $p(\rho)$, is obtained as follows (Narain et al. 2007),

$$
\begin{gathered}
\rho=\frac{1}{\pi^{2}} \int_{0}^{k_{F}} k^{2} \sqrt{m^{2}+k^{2}} d k=\frac{m^{4}}{8 \pi^{2}}\left[\left(2 z^{3}+z\right)\left(1+z^{2}\right)^{1 / 2}-\sinh ^{-1}(z)\right], \\
p=\frac{1}{3 \pi^{2}} \int_{0}^{k_{F}} \frac{k^{4}}{\sqrt{m^{2}+k^{2}}} d k=\frac{m^{4}}{24 \pi^{2}}\left[\left(2 z^{3}-3 z\right)\left(1+z^{2}\right)^{1 / 2}+3 \sinh ^{-1}(z)\right] .
\end{gathered}
$$

In the above equation, $k_{F}=\left(3 \pi^{2} n\right)^{1 / 3}$ denotes the Fermi momentum and $\mathrm{n}$ is the total number density of fermions. In addition, $m$ shows the mass of fermions and $z=k_{F} / m$. The EOS with mass $m=1.0 \mathrm{GeV}$ for the fermions is presented in Figure 3.

In this work, we study a DM admixed neutron star with two concentric spheres: one containing the NM and the other the DM. The EOS of NM in the NM sphere is presented in Figure 1, and the EOSs of DM in the DM sphere are given in Figures 2 and 3. In our calculations for the NM sphere, in the crust at densities lower than $0.05 \mathrm{fm}^{-3}$, we use the EOS calculated by Baym et al. (1971). The contribution of DM in the DMANS can be different and depends on the history of the star (Sandin \& Ciarcelluti 2009) and the way that it originates (Xiang et al. 2014). The gravitational field of the normal star in all phases of evolution can affect the DM and capture it into the star. This is more probable when the star is more compact. In contrast, the opposite scenario in which the DM objects trap the baryons has been also proposed (Ciarcelluti \& Sandin 2011). In this case, a more significant contribution of DM in the DMANS is expected. In this study, we explore DMANSs with different proportions of DM in the center of the DMANS. This will be done by considering different central pressure ratios, i.e., central DM pressure to central NM pressure ratio $\delta=\frac{p_{D}(r=0)}{p_{N}(r=0)}$, for the DMANS. The zero pressure ratio, $\delta=0$, shows a normal neutron star.

In our calculations of the DMANS structure, we apply the two-fluid formalism for the NM and DM presented by (Sandin \& Ciarcelluti 2009; Ciarcelluti \& Sandin 2011). In this formalism, it is proposed that DM interacts with NM just through gravity. One static and spherically symmetric space-time with the line element, (we use the units in 


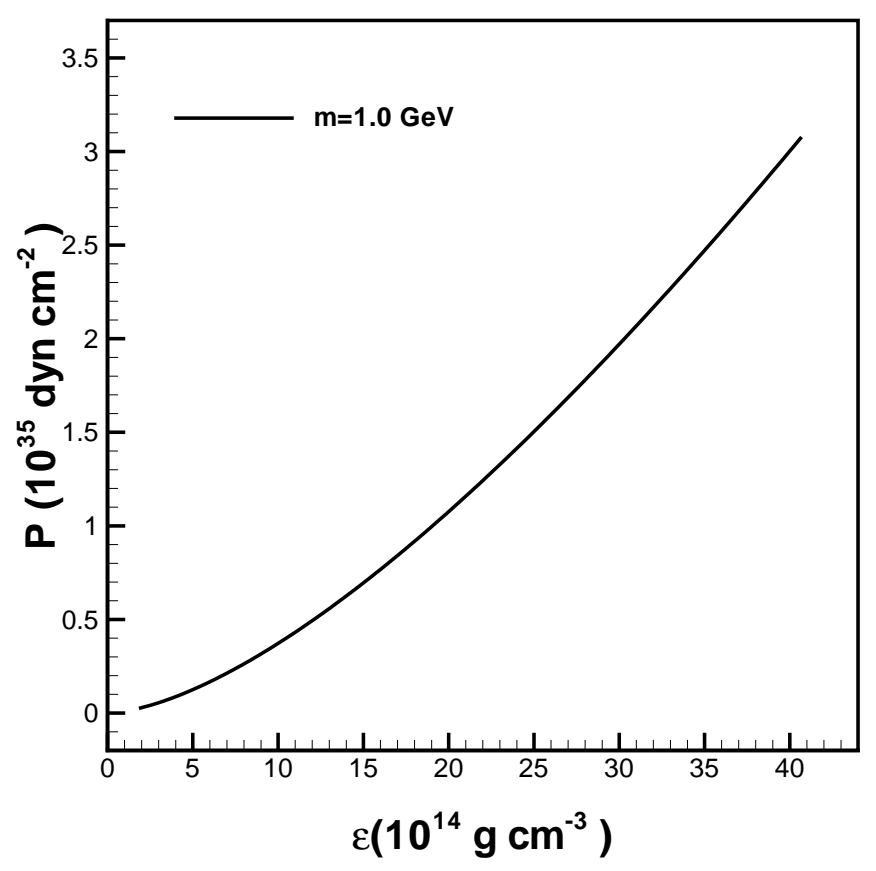

Figure 3. Equation of state for a free gas of fermions at zero temperature with the value of $m=1.0 \mathrm{GeV}$ for the mass of fermions.

which $\mathrm{G}=\mathrm{c}=1$ ),

$$
d \tau^{2}=e^{2 \nu(r)} d t^{2}-e^{2 \lambda(r)} d r^{2}-r^{2}\left(d \theta^{2}+\sin ^{2} \theta d \phi^{2}\right),
$$

and the energy-momentum tensor of a perfect fluid,

$$
T^{\mu \nu}=-p g^{\mu \nu}+(p+\varepsilon) u^{\mu} u^{\nu},
$$

are considered. In the above equation, $p$ and $\varepsilon$ are the total pressure and total energy density, respectively. The total energy density is related to the rest-mass energy density $\left(\varepsilon_{r m}\right)$ and internal energy density $\left(\varepsilon_{i n}\right)$ by

$$
\varepsilon=\varepsilon_{r m}+\varepsilon_{i n} .
$$

In our system, the total pressure and total energy density are the results of both NM and DM quantities,

$$
\begin{aligned}
& p(r)=p_{N}(r)+p_{D}(r), \\
& \varepsilon(r)=\varepsilon_{N}(r)+\varepsilon_{D}(r),
\end{aligned}
$$

in which $p_{i}$ and $\varepsilon_{i}$ are the pressure and energy density of neutron $(i=N)$ and dark $(i=D)$ matter at position $r$ from the center of the star, respectively. By applying some calculations, the Einstein field equations lead to (Sandin \& Ciarcelluti 2009; Ciarcelluti \& Sandin 2011; Xiang et al. 2014),

$$
\begin{gathered}
e^{-2 \lambda(r)}=1-\frac{2 M(r)}{r}, \\
\frac{d \nu}{d r}=\frac{M(r)+4 \pi r^{3} p(r)}{r[r-2 M(r)]}, \\
\frac{d p_{N}}{d r}=-\left[p_{N}(r)+\varepsilon_{N}(r)\right] \frac{d \nu}{d r}, \\
\frac{d p_{D}}{d r}=-\left[p_{D}(r)+\varepsilon_{D}(r)\right] \frac{d \nu}{d r} .
\end{gathered}
$$




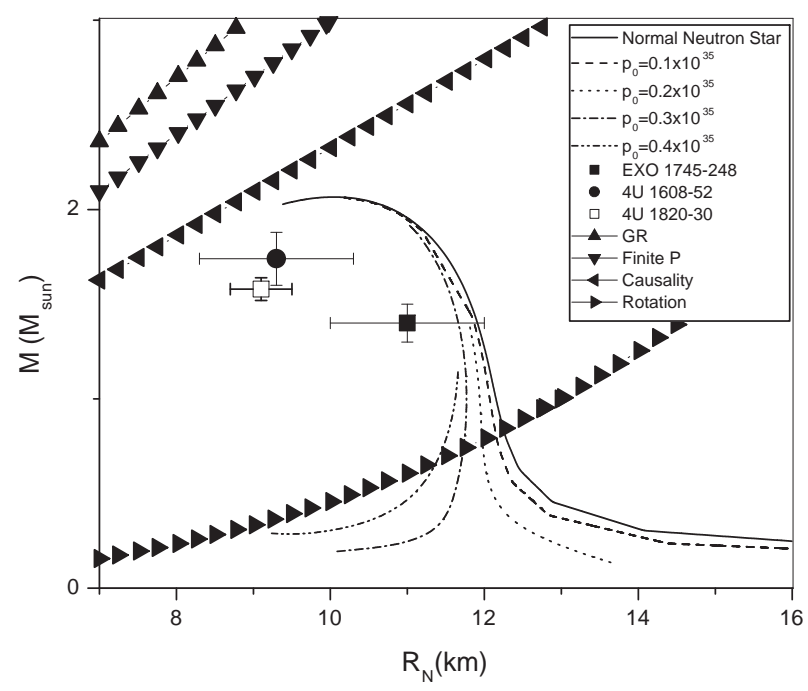

Figure 4. $M-R_{N}$ relation for a normal neutron star and DMANSs with different values of $p_{0}$ in units of $d y n \mathrm{~cm}^{-2}$ for the case of $\delta=1$ and some observational data. Curves that exclude some regions, i.e., by the general relativity $M>\frac{c^{2} R}{2 G}(\mathrm{GR})$, by the finite pressure $M>\frac{4}{9} \frac{c^{2} R}{G}$ (Finite P), by causality $M>\frac{10}{29} \frac{c^{2} R}{G}$ (Causality), and by the rotation of 716 Hz pulsar J1748-2446ad (Rotation), are also presented (Lattimer \& Prakash 2007).

In the above equations, $r$ denotes the radial coordinate from the center of the star. $M(r)=\int_{0}^{r} d r 4 \pi r^{2} \varepsilon(r)$ is the total mass inside a sphere with radius $r$. This separation for DM and NM is due to the assumption that the two sectors interact just via gravity. The structure of DMANS is described by these two-fluid TOV equations. The condition for the DMANS radius, $R$, and mass, $M(R)$, is $p(R)=0$ (Xiang et al. 2014). Moreover, the radius and mass of the NM sphere and DM sphere are determined with the conditions $p_{N}\left(R_{N}\right)=0$ and $p_{D}\left(R_{D}\right)=0$, respectively (Xiang et al. 2014). It should be noted that the radial dependencies of the pressure and density are different in the two sectors.

\section{RESULTS AND DISCUSSION}

Figures 4-7 show the total mass of the DMANS versus the radius of the NM sphere $\left(M-R_{N}\right.$ relation) for a normal neutron star and DMANSs with different DM equations of state (DMEOSs) when the central pressures of NM and DM are the same, i.e., $\delta=1$. The curves that apply constraints for the mass and radius, i.e., by the general relativity $M>\frac{c^{2} R}{2 G}(\mathrm{GR})$, by the finite pressure $M>\frac{4}{9} \frac{c^{2} R}{G}$ (Finite P), by causality $M>\frac{10}{29} \frac{c^{2} R}{G}$ (Causality), and by the rotation of $716 \mathrm{~Hz}$ pulsar J1748-2446ad (Rotation), are also presented (Lattimer \& Prakash 2007). The mass-radius relations that pass in the permitted region are acceptable. We can see that with all DMEOSs the radius of a DMANS is smaller than the radius of a normal neutron star with the same mass. This decrease of radius in DMANSs is in agreement with previous results (Leung \& Chu \& Lin 2011, 2012; Xiang et al. 2014). $\quad$ It is obvious that the $M-R_{N}$ relations obtained using DMEOSs corresponding to $p_{0} \leq 0.2 \times 10^{35} \mathrm{dyn} \mathrm{cm}^{-2}$ show a behavior like that of normal neutron stars that are gravitationally bound. However, the other DMEOSs, which are the stiffer equations of state, lead to $M-R_{N}$ relations that are significantly different from the normal neutron stars. Such $M-R_{N}$ relations with different slopes, representing the self-bound DMANSs, are the result of the interaction between DM and NM. The significant effects of the stiff DM equations of state on $M-R_{N}$ relations have been also mentioned in Xiang et al. (2014). Our results indicate that the $M-R_{N}$ relation for the DMEOS with $p_{0}=0.5 \times 10^{35} \mathrm{dyn} \mathrm{cm}^{-2}$ is approximately in agreement with the result related to the DMEOS with $m=1.0 \mathrm{GeV}$. Figures 4-7 show that, for some $M-R_{N}$ relations, with a value of $R_{N}$ two possible DMANSs with different masses can exist. This is a result of different degrees of DM domination in DMANSs. Put differently, two equal sized DMANSs that are affected by the DM differently have different masses.

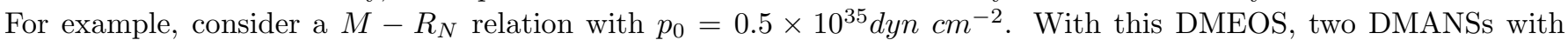
similar radii 11.25 and $11.36 \mathrm{~km}$ have masses of about $0.77 M_{\odot}$ and $1.67 M_{\odot}$, respectively. The ratios of the mass of the DM sphere to the total mass for these two DMANSs are $\frac{M_{D}}{M}=0.13$ and $\frac{M_{D}}{M}=0.02$ respectively, indicating the greater effect of DM in the former. In addition, in the case of $m=1.0 \mathrm{GeV}$, two DMANSs with radii of 10.41 and $10.39 \mathrm{~km}$ have masses of $0.41 M_{\odot}$ and $1.95 M_{\odot}$, and the ratios of $\frac{M_{D}}{M}$ are 0.29 and 0.03 , respectively. The maximum mass of DMANS depends on the DMEOSs (see Table 1). We can see that with all DMEOSs, the maximum mass of the 


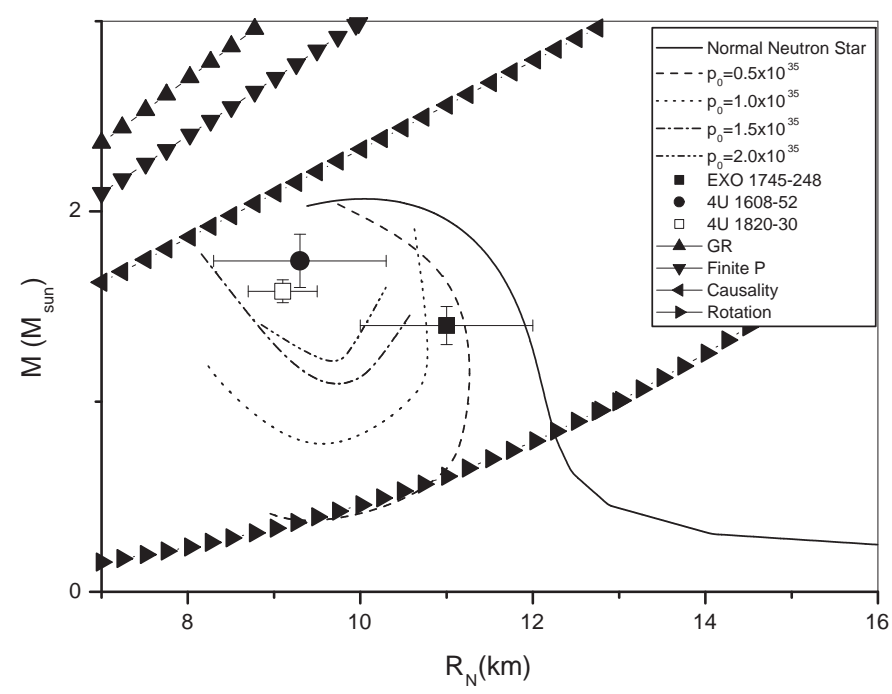

Figure 5. Same as Figure 4 but for other values of $p_{0}$ in $d y n \mathrm{~cm}^{-2}$ unit.

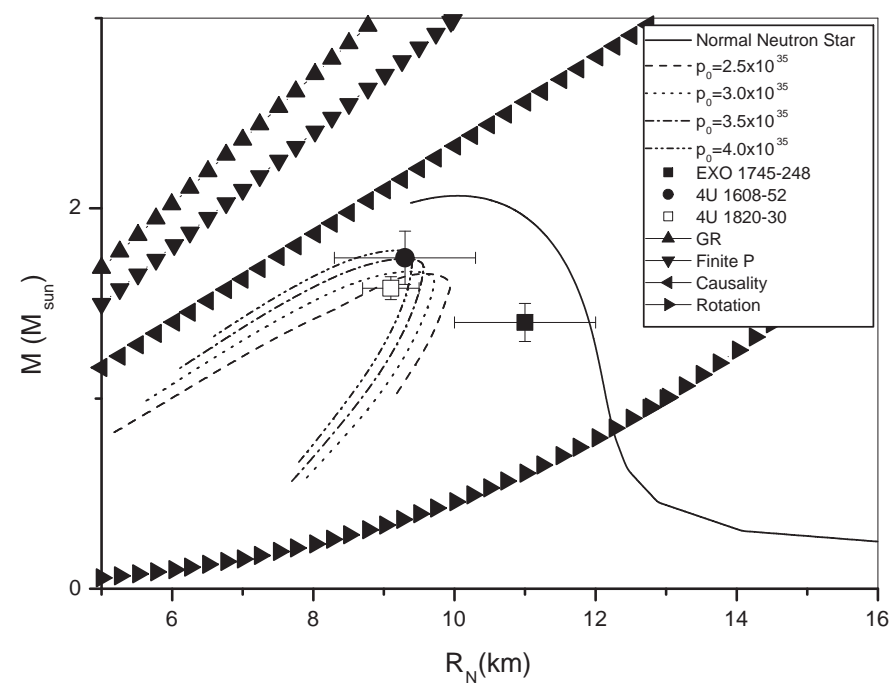

Figure 6. Same as Figure 4 but for other values of $p_{0}$ in $d y n \mathrm{~cm}^{-2}$ unit.

DMANS is lower than that of the normal neutron star. The decrease of the maximum mass due to the DM has been also reported in other studies (Leung \& Chu \& Lin 2011, 2012; Li \& Huang \& Xu 2012; Xiang et al. 2014). Figures 4-7 also show the comparison of observational data with the theoretical results. It is obvious that EXO 1745-248, with mass $M=1.4 \pm 0.1 M_{\odot}$ and radius $R=11 \pm 1 \mathrm{~km}$, can hardly be modeled with the normal neutron star. However,

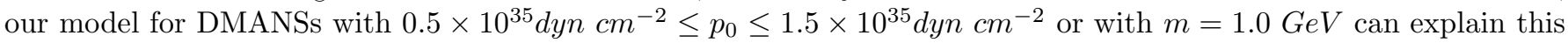
observational result. The neutron star in $4 \mathrm{U} 1820-30$ with $M=1.58 \pm 0.06 M_{\odot}$ and $R=9.1 \pm 0.4 \mathrm{~km}$ and the neutron star in $4 \mathrm{U} 1608-52$ with $M=1.74 \pm 0.14 M_{\odot}$ and $R=9.3 \pm 1.0 \mathrm{~km}$ cannot be normal neutron stars. It is clear that the DMEOSs with $2.5 \times 10^{35} \mathrm{dyn} \mathrm{cm}^{-2} \leq p_{0} \leq 4.0 \times 10^{35} \mathrm{dyn}_{\mathrm{cm}}^{-2}$ corresponding to self-bound stars lead to results that are in agreement with the properties of the neutron stars in $4 \mathrm{U}$ 1608-52 and $4 \mathrm{U} 1820-30$.

Table 2 presents the mass and radius of the dark and NM spheres relating to the DMANSs with the maximum mass. It can be seen that for DMANSs with DMEOSs corresponding to $p_{0} \leq 1.0 \times 10^{35} \mathrm{dyn}_{\mathrm{cm}}^{-2}$, the significant contribution in the mass is related to the NM sphere. In addition, in these cases, the radius of the DM sphere is smaller than the 


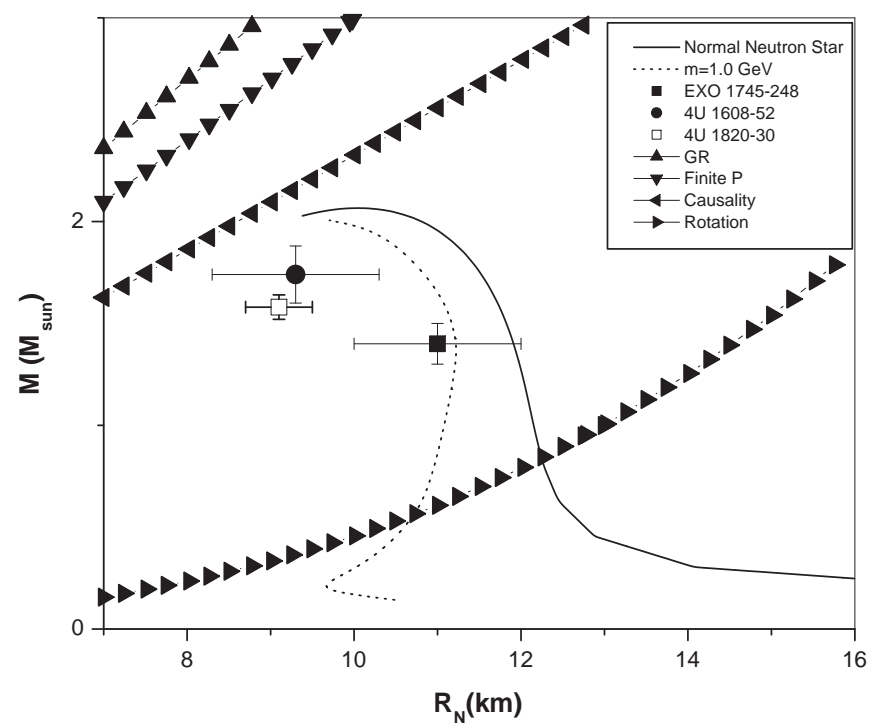

Figure 7. Same as Figure 4 but for the DMANS with the DMEOS given in Figure 3.

Table 1. The Maximum Masses and Corresponding Visible Radii for A Normal Neutron star and DMANSs with Different Dark Matter Equations of State for the Case of $\delta=1$

\begin{tabular}{|c|c|c|}
\hline & $M_{\max }\left(M_{\odot}\right)$ & $\mathrm{R}(\mathrm{km})$ \\
\hline Normal Neutron Star & 2.07 & 10.04 \\
\hline$p_{0}\left(10^{35}{\left.\text { dyn } \mathrm{cm}^{-2}\right)}^{-2}\right.$ & & \\
0.1 & 2.06 & 10.18 \\
0.2 & 1.39 & 11.80 \\
0.3 & 1.92 & 11.02 \\
0.4 & 1.16 & 11.66 \\
0.5 & 2.05 & 9.71 \\
1.0 & 1.91 & 10.63 \\
1.5 & 1.78 & 8.16 \\
2.0 & 1.60 & 10.30 \\
2.5 & 1.68 & 9.35 \\
3.0 & 1.68 & 9.38 \\
3.5 & 1.74 & 9.25 \\
4.0 & 1.78 & 9.11 \\
\hline $\mathrm{m}(\mathrm{GeV})$ & & \\
1.0 & 2.01 & 9.70 \\
\hline
\end{tabular}

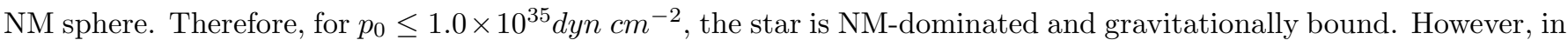

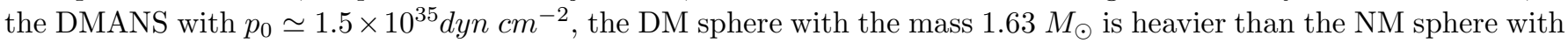
the mass $0.15 M_{\odot}$. In this star, the DM halo of radius $19.95 \mathrm{~km}$ surrounds the NM sphere of smaller radius $8.16 \mathrm{~km}$. This is a DM-dominated neutron star. We can conclude from the above discussion that the neutron star EXO 1745-248 could be a DM-dominated neutron star. Figures 5 and 7 indicate that both DMEOSs $p_{0} \simeq 1.0 \times 10^{35} \mathrm{dyn}_{\mathrm{cm}}^{-2}$ and $m=1.0 \mathrm{GeV}$, which lead to self-bound DMANSs, can be used to explain the observational result of EXO 1745-248. Moreover, Table 2 shows that the masses of a DM sphere obtained from these two models, i.e., $0.06 M_{\odot}$ and $0.05 M_{\odot}$ respectively, are approximately the same. In addition, the DMEOS with $p_{0} \simeq 2.5 \times 10^{35} \mathrm{dyn} \mathrm{cm}^{-2}$ leads to a DMANS with a heavier and larger DM sphere compared to the NM sphere. Consequently, this star is also a DM-dominated neutron star. Therefore, the neutron stars $4 \mathrm{U}$ 1608-52 and $4 \mathrm{U}$ 1820-30 could be self-bound and DM-dominated neutron 
Table 2. The Mass and Radius of the Dark and Neutron Matter Spheres of the Maximum Mass DMANSs with Different DMEOSs for the Central Pressure Ratio $\delta=1$

\begin{tabular}{|c|c|c|c|c|c|}
\hline$p_{0}\left(10^{35}\right.$ dyn cm $\left.\mathrm{cm}^{-2}\right)$ & $M_{D}\left(M_{\odot}\right)$ & $R_{D}(\mathrm{~km})$ & $M_{N}\left(M_{\odot}\right)$ & $R_{N}(\mathrm{~km})$ & $M_{\max }\left(M_{\odot}\right)$ \\
\hline 0.1 & $<0.01$ & 1.85 & 2.06 & 10.18 & 2.06 \\
0.2 & 0.02 & 4.34 & 1.37 & 11.80 & 1.39 \\
0.3 & 0.01 & 3.48 & 1.90 & 11.02 & 1.92 \\
0.4 & 0.05 & 5.86 & 1.11 & 11.66 & 1.16 \\
0.5 & 0.01 & 2.70 & 2.04 & 9.71 & 2.05 \\
1.0 & 0.06 & 5.16 & 1.85 & 10.63 & 1.91 \\
1.5 & 1.63 & 19.95 & 0.15 & 8.16 & 1.78 \\
2.0 & 0.24 & 8.35 & 1.36 & 10.30 & 1.60 \\
2.5 & 0.99 & 15.92 & 0.69 & 9.35 & 1.68 \\
3.0 & 0.34 & 9.38 & 1.34 & 9.38 & 1.68 \\
3.5 & 0.34 & 9.25 & 1.40 & 9.25 & 1.74 \\
4.0 & 0.34 & 9.11 & 1.44 & 9.11 & 1.78 \\
\hline$m(\mathrm{GeV})$ & & & & & \\
1.0 & 0.05 & 3.26 & 1.96 & 9.70 & 2.01 \\
\hline
\end{tabular}
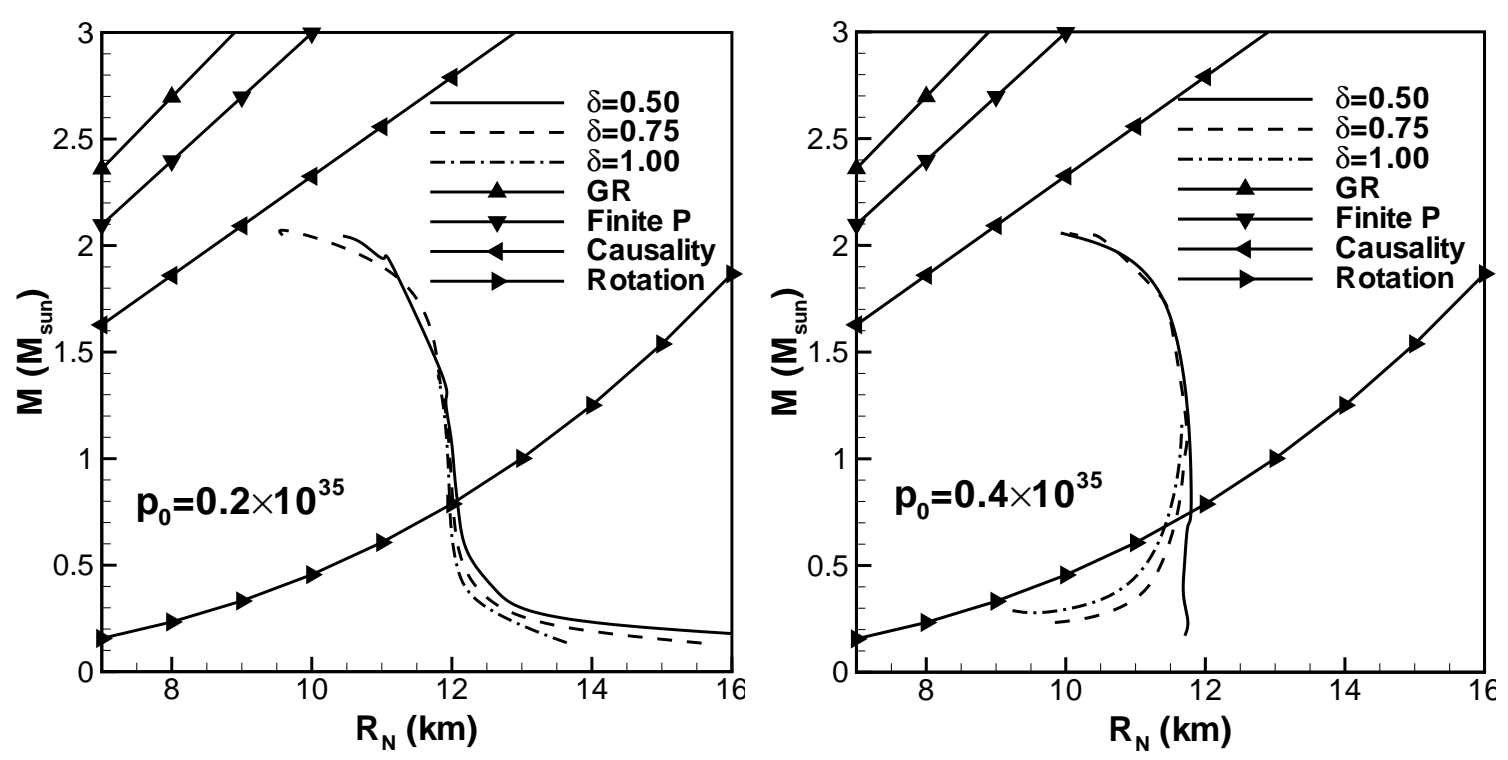

Figure 8. $M-R_{N}$ relations and constraint curves with two values of $p_{0}$ in units of $d y n \mathrm{~cm}^{-2}$ for different central pressure ratios, $\delta$.

stars. Table 2 indicates that the DMANSs with DMEOSs corresponding to $p_{0} \geq 3.0 \times 10^{35} \mathrm{dyn}_{\mathrm{cm}}^{-2}$ are stars with a more massive NM sphere, but have two spheres of equal radius. By increasing the value of the free parameter $p_{0}$ from 3.0 to 4.0, the mass of the $\mathrm{NM}$ sphere increases while the mass of the DM sphere remains constant at $0.34 M_{\odot}$. The increase in the value of $p_{0}$ reduces the radius of the NM and DM spheres, leading to the compactness of the star.

Figures 8-10 show the $M-R_{N}$ relations with different DMEOSs for various central pressure ratios, $\delta$. It is obvious that, for all DMEOSs, when the central pressure ratio grows from 0.5 to 1 , the low mass DMANSs (about $M \leq M_{\odot}$ ) will become more compact. This effect is more significant for the case of $m=1.0 \mathrm{GeV}$. In addition, by increasing the central pressure ratio from 2 to 5 , for DMANSs with $p_{0}=0.2 \times 10^{35} \mathrm{dyn}_{\mathrm{cm}}^{-2}$ the radius of the star is not

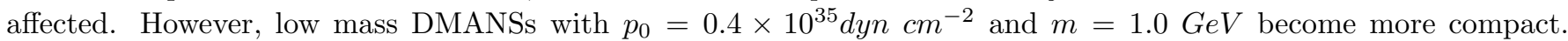
DMANSs with $m=1.0 \mathrm{GeV}$ are more affected by the value of central pressure ratio compared to the cases with

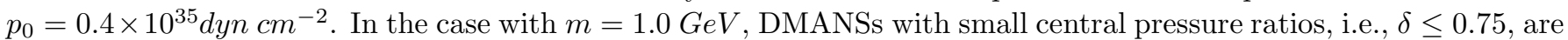
like normal neutron stars and gravitationally bound. However, for higher values of central pressure ratio the DMANSs are self-bound stars. We have found from Figure 9 that DMANSs with the DMEOS $p_{0}=0.2 \times 10^{35} \mathrm{dyn}_{\mathrm{cm}}^{-2}$ and 

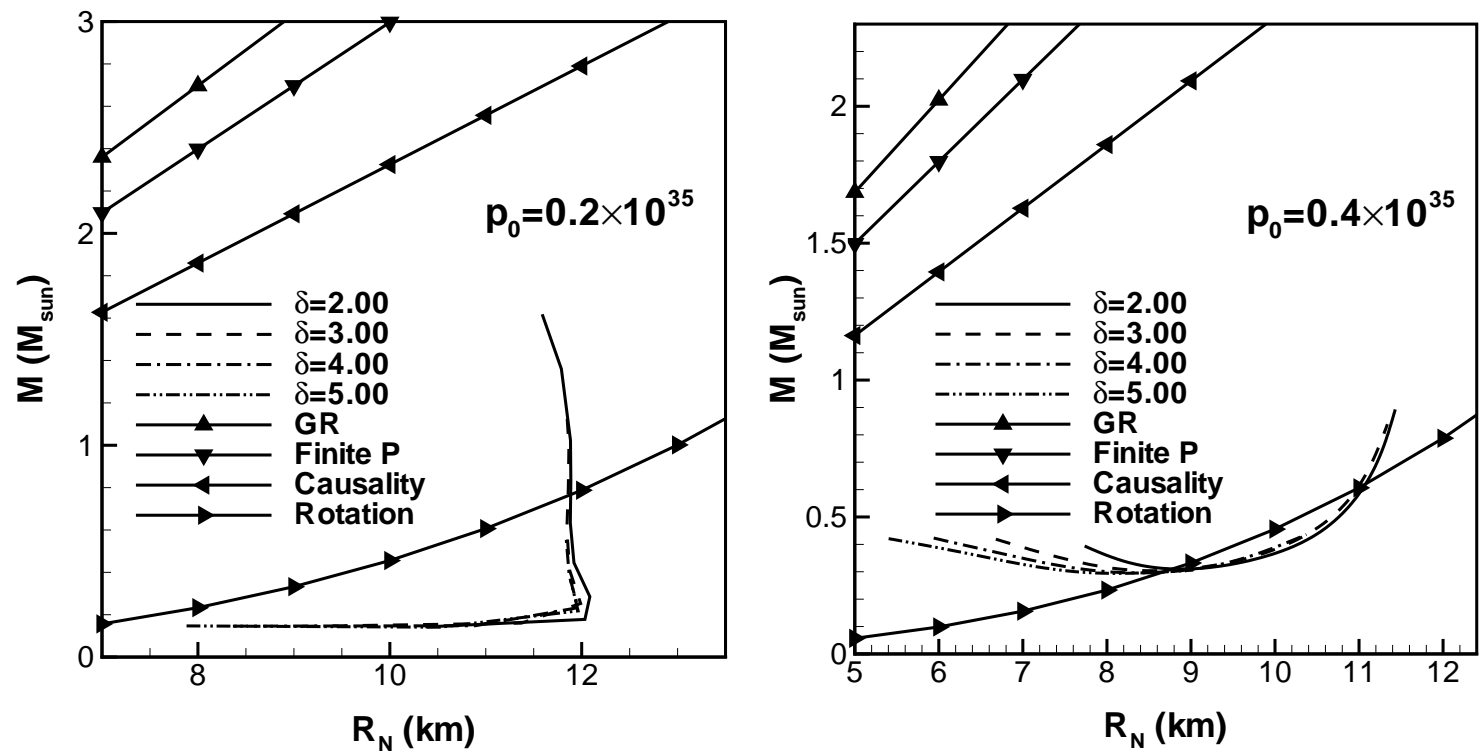

Figure 9. Same as Figure 8 but for other values of central pressure ratio, $\delta$.
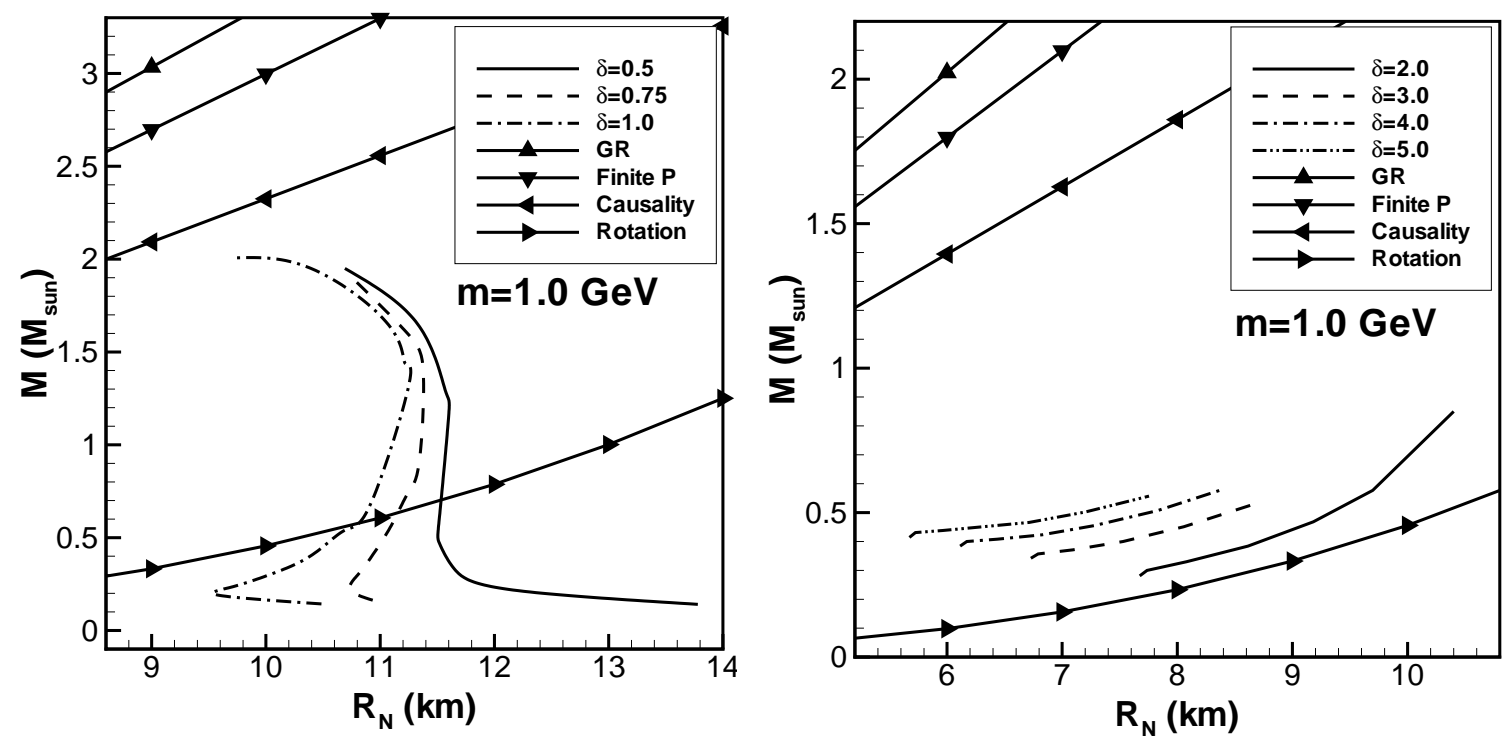

Figure 10. $M-R_{N}$ relations and constraint curves with the fermionic DMEOS for different central pressure ratios, $\delta$.

central pressure ratios $\delta=4.00$ and $\delta=5.00$ do not exist. Figures 8-10 confirm that the $M-R_{N}$ relation can be influenced significantly by the central pressure ratio, similar to the results of (Xiang et al. 2014). The results for the maximum masses and corresponding radii are presented in Tables 3-5. It can be seen from Tables 3 and 4 that, for DMANSs with $p_{0}=0.2 \times 10^{35} \mathrm{dyn} \mathrm{cm}^{-2}$ and $\delta \geq 2$ as well as the DMANSs with $p_{0}=0.4 \times 10^{35} \mathrm{dyn}_{\mathrm{cm}}^{-2}$, the maximum mass decreases by increasing the central pressure ratio, in agreement with the results of (Xiang et al. 2014). Table 5 also indicates that, in the case of fermionic DMEOS, $m=1.0 \mathrm{GeV}$, the maximum masses are smaller for $\delta>1$ than the cases with $\delta<1$.

The total mass versus radius of the NM and DM spheres for different DMEOSs and various values of central pressure ratio is given in Figures 11-14. These figures show the size of the NM and DM spheres for a DMANS with a specific mass. We can see that, for all values of central pressure ratio, the radius of the NM sphere of the star with $p_{0}=0.4 \times 10^{35} \mathrm{dyn} \mathrm{cm}^{-2}$ is smaller than the case of $p_{0}=0.2 \times 10^{35} \mathrm{dyn}_{\mathrm{cm}}^{-2}$ with the same mass. However,

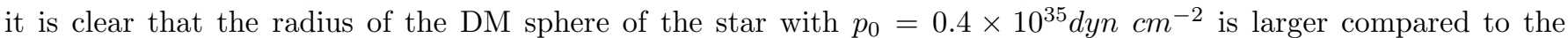
case with $p_{0}=0.2 \times 10^{35} \mathrm{dyn} \mathrm{cm}^{-2}$. Figures 11-14 indicate that in the cases with $p_{0}=0.2 \times 10^{35} \mathrm{dyn}_{\mathrm{cm}}^{-2}$ and 
Table 3. The Maximum Masses and Corresponding Visible Radii for DMANSs with DMEOS Corresponding to $p_{0}=0.2 \times$

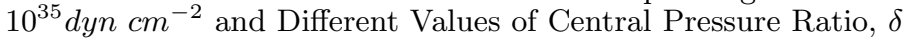

\begin{tabular}{|c|c|c|}
\hline$\delta$ & $M_{\max }\left(M_{\odot}\right)$ & $R(\mathrm{~km})$ \\
\hline 0.50 & 2.05 & 10.44 \\
0.75 & 2.06 & 9.81 \\
1.00 & 1.39 & 11.80 \\
2.00 & 1.62 & 11.59 \\
3.00 & 1.18 & 11.84 \\
\hline
\end{tabular}

Note. In this case, DMANSs with $\delta \geq 4.00$ do not exist.

Table 4. Same as Table 3 but for DMANSs with DMEOS Corresponding to $p_{0}=0.4 \times 10^{35} \mathrm{dyn}_{\mathrm{cm}}^{-2}$

\begin{tabular}{|c|c|c|}
\hline$\delta$ & $M_{\max }\left(M_{\odot}\right)$ & $R(\mathrm{~km})$ \\
\hline 0.50 & 2.06 & 9.93 \\
0.75 & 2.06 & 10.05 \\
1.00 & 1.16 & 11.66 \\
2.00 & 0.89 & 11.43 \\
3.00 & 0.84 & 11.34 \\
4.00 & 0.42 & 5.94 \\
5.00 & 0.42 & 5.40 \\
\hline
\end{tabular}

Table 5. Same as Table 3 but for DMANSs with Fermionic DMEOS Corresponding to $m=1.0 \mathrm{GeV}$

\begin{tabular}{|c|c|c|}
\hline$\delta$ & $M_{\max }\left(M_{\odot}\right)$ & $R(\mathrm{~km})$ \\
\hline 0.50 & 1.95 & 10.69 \\
0.75 & 1.88 & 10.79 \\
1.00 & 2.01 & 9.70 \\
2.00 & 0.85 & 10.40 \\
3.00 & 0.53 & 8.65 \\
4.00 & 0.58 & 8.41 \\
5.00 & 0.56 & 7.75 \\
\hline
\end{tabular}

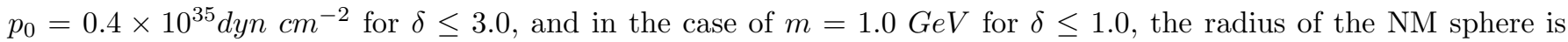
larger than the radius of the DM one. The difference between the radius of the NM and DM spheres is larger in stars with $p_{0}=0.2 \times 10^{35} \mathrm{dyn} \mathrm{cm}^{-2}$ compared to the case of $p_{0}=0.4 \times 10^{35} \mathrm{dyn} \mathrm{cm}^{-2}$. For all DMEOSs, by increasing the central pressure ratio, the radius of the NM sphere decreases while the radius of the DM sphere increases. For stars with $p_{0}=0.4 \times 10^{35} \mathrm{dyn} \mathrm{cm}^{-2}$ and $\delta \geq 3.00$, as well as stars with $m=1.0 \mathrm{GeV}$ and $\delta \geq 2.00$, we can see that there are low mass DMANSs in which the NM sphere is surrounded by a halo of DM, which is consistent with previous results (Leung \& Chu \& Lin 2011, 2012). It is evident that with the higher values of $\delta$ (for example in stars with

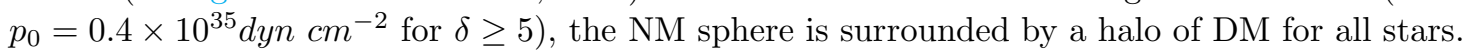

Tables 6-8 present the mass and radius of the dark and NM spheres of the maximum mass DMANSs with different DMEOSs and for various values of central pressure ratio. We can see that the mass of a DM sphere increases when the central pressure ratio increases. This effect is more significant in the case of $p_{0}=0.4 \times 10^{35} \mathrm{dyn}_{\mathrm{cm}}^{-2}$. In addition, with this DMEOS the mass of the NM sphere decreases as the central pressure ratio grows. In the case with

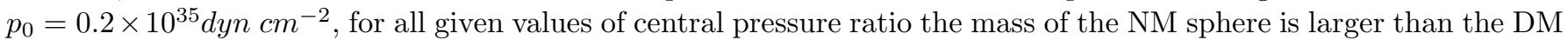
sphere. Therefore, the DMANSs are NM-dominated in these cases. However, for stars with $p_{0}=0.4 \times 10^{35} \mathrm{dyn}_{\mathrm{cm}}^{-2}$ 

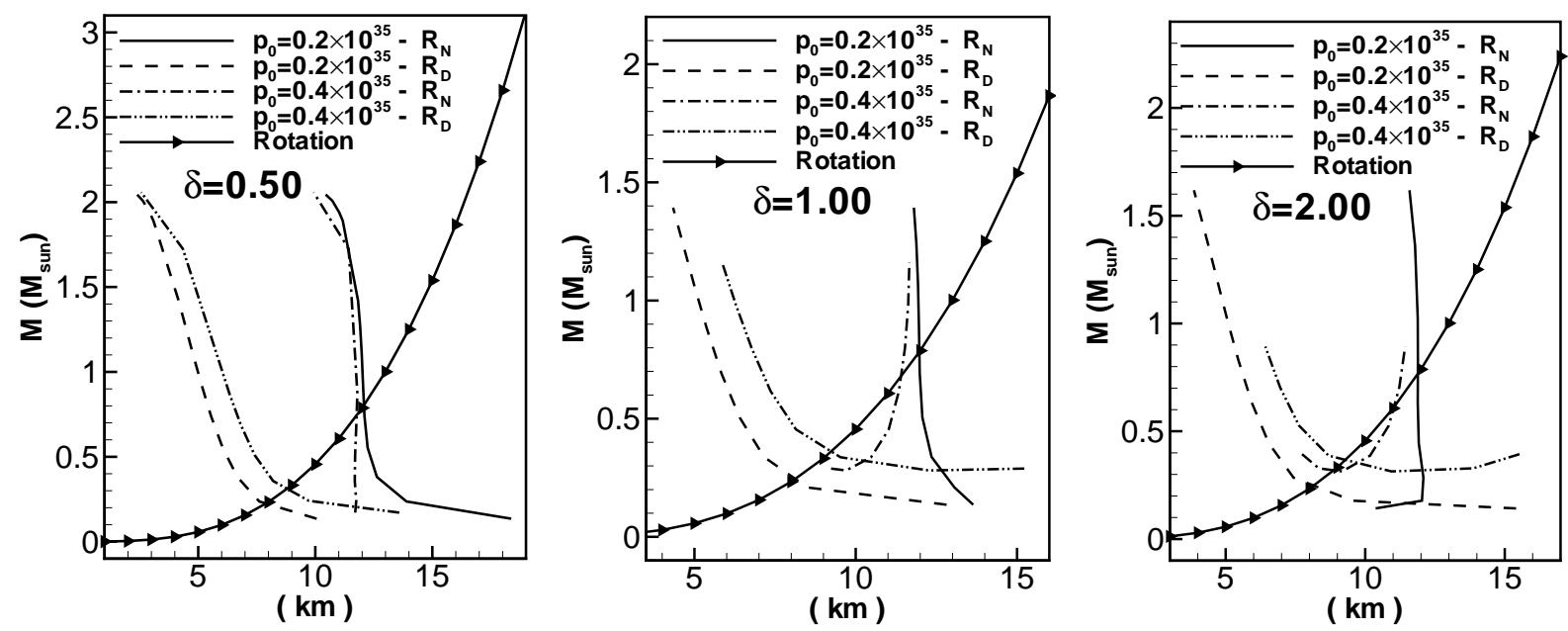

Figure 11. The total mass vs. neutron $\left(R_{N}\right)$ and dark $\left(R_{D}\right)$ matter sphere radii of DMANSs, and the curve that excludes a region by the rotation of $716 \mathrm{~Hz}$ pulsar J1748-2446ad (Rotation), with different values of $p_{0}$ in units of $d y n \mathrm{~cm}^{-2}$ and central pressure ratio, $\delta$.
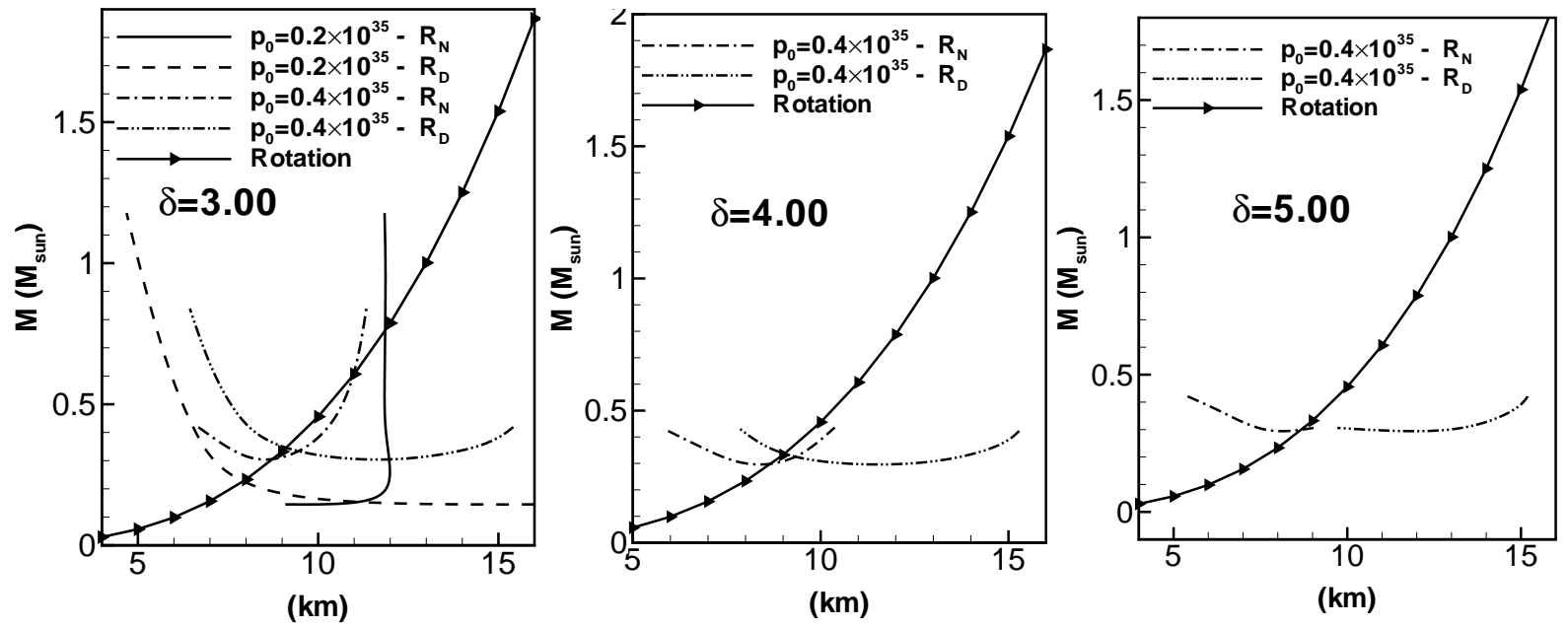

Figure 12. Same as Figure 11 but for other values of central pressure ratio, $\delta$.

Table 6. The Mass and Radius of the Dark and Neutron Matter Spheres of the Maximum Mass DMANSs with DMEOS

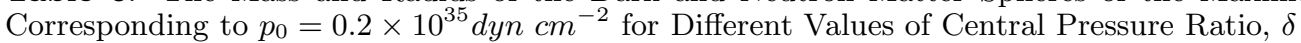

\begin{tabular}{|c|c|c|c|c|c|}
\hline$\delta$ & $M_{D}\left(M_{\odot}\right)$ & $R_{D}(k m)$ & $M_{N}\left(M_{\odot}\right)$ & $R_{N}(k m)$ & $M_{\max }\left(M_{\odot}\right)$ \\
\hline 0.50 & 0.01 & 2.39 & 2.04 & 10.44 & 2.05 \\
0.75 & 0.01 & 1.96 & 2.05 & 9.811 & 2.06 \\
1.00 & 0.02 & 4.34 & 1.37 & 11.80 & 1.39 \\
2.00 & 0.02 & 3.86 & 1.60 & 11.59 & 1.62 \\
3.00 & 0.03 & 4.69 & 1.15 & 11.84 & 1.18 \\
\hline
\end{tabular}

and $\delta \geq 4$, as well as the stars with $m=1.0 \mathrm{GeV}$ and $\delta=5.00$, the DM sphere is heavier than the NM one. Thus, these stars are DM-dominated. It is obvious from Tables 6-8 that for the neutron dominated DMANSs the halo of DM is confined within the NM sphere. In contrast, in the DM dominated DMANSs the NM sphere is located inside the halo of DM which is in agreement with other investigations (Leung \& Chu \& Lin 2011, 2012).

Considering two stars with the same mass-one of them a normal neutron star and the other a DMANS in which the Nm sphere is surrounded by the halo of DM-they have the same gravitational effects owing to their equal mass. 

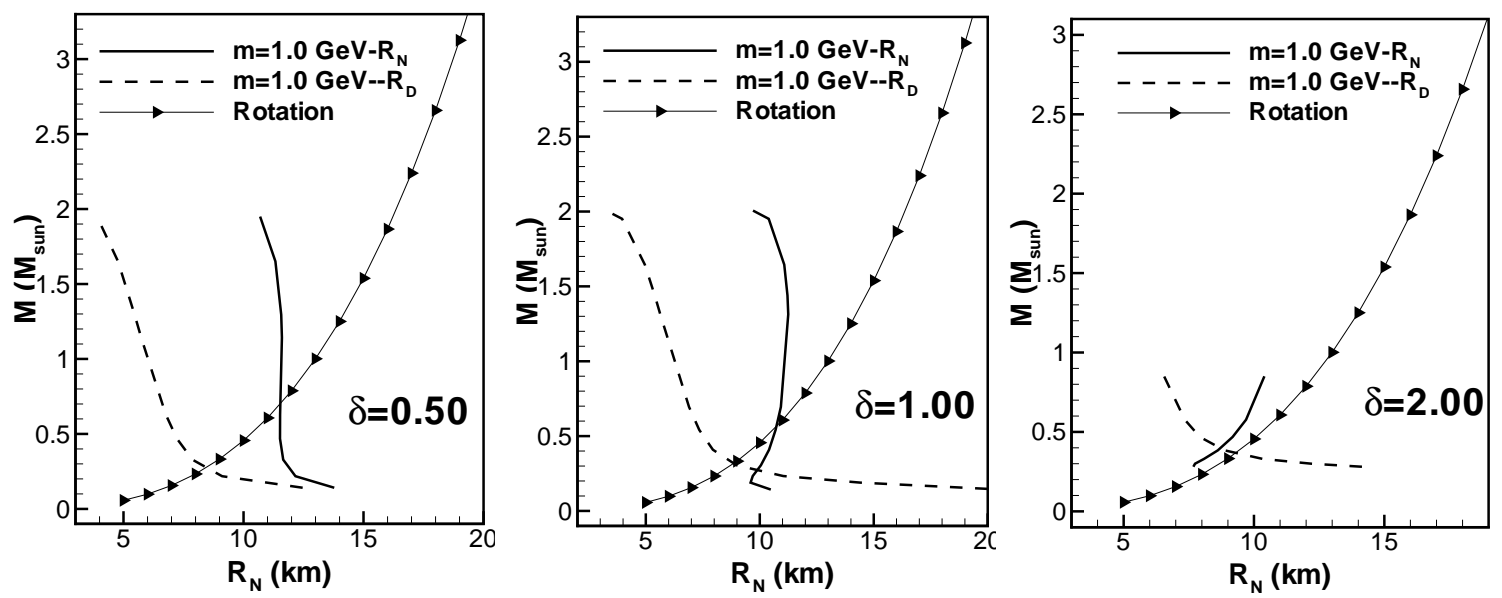

Figure 13. The total mass vs. neutron $\left(R_{N}\right)$ and dark $\left(R_{D}\right)$ matter sphere radii of DMANSs, and the curve that excludes a region by the rotation with the fermionic DMEOS for different central pressure ratios, $\delta$.
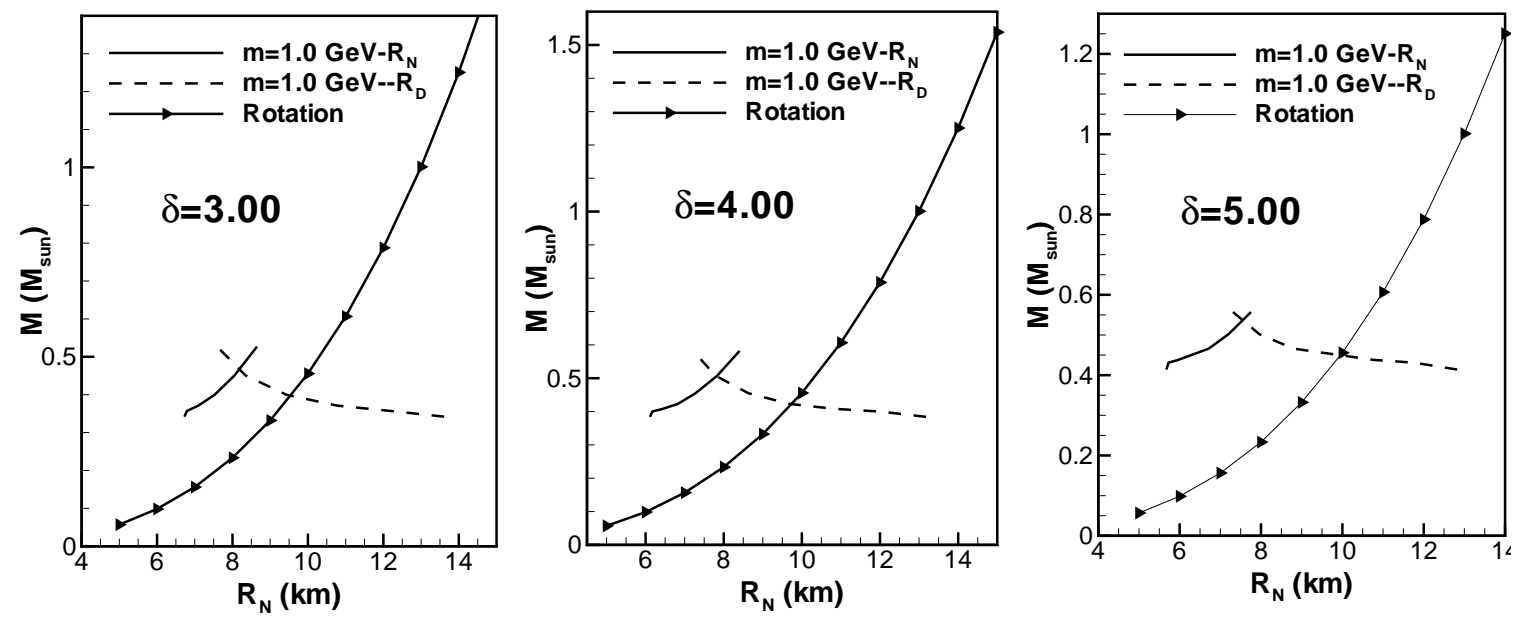

Figure 14. Same as Figure 13 but for other values of central pressure ratio, $\delta$.

Table 7. Same as Table 6 but for DMANSs with DMEOS Corresponding to $p_{0}=0.4 \times 10^{35} \mathrm{dyn}_{\mathrm{cm}}^{-2}$

\begin{tabular}{|c|c|c|c|c|c|}
\hline$\delta$ & $M_{D}\left(M_{\odot}\right)$ & $R_{D}(k m)$ & $M_{N}\left(M_{\odot}\right)$ & $R_{N}(k m)$ & $M_{\max }\left(M_{\odot}\right)$ \\
\hline 0.50 & 0.01 & 2.56 & 2.05 & 9.93 & 2.06 \\
0.75 & 0.01 & 2.75 & 2.05 & 10.05 & 2.06 \\
1.00 & 0.05 & 5.86 & 1.11 & 11.66 & 1.16 \\
2.00 & 0.08 & 6.42 & 0.81 & 11.43 & 0.89 \\
3.00 & 0.08 & 6.44 & 0.76 & 11.34 & 0.84 \\
4.00 & 0.41 & 15.29 & 0.01 & 5.94 & 0.42 \\
5.00 & 0.41 & 15.19 & 0.01 & 5.40 & 0.42 \\
\hline
\end{tabular}


Table 8. Same as Table 6 but for DMANSs with fermionic DMEOS corresponding to $m=1.0 \mathrm{GeV}$.

\begin{tabular}{|c|c|c|c|c|c|}
\hline$\delta$ & $M_{D}\left(M_{\odot}\right)$ & $R_{D}(k m)$ & $M_{N}\left(M_{\odot}\right)$ & $R_{N}(k m)$ & $M_{\max }\left(M_{\odot}\right)$ \\
\hline 0.50 & 0.04 & 3.90 & 1.91 & 10.69 & 1.95 \\
0.75 & 0.06 & 4.27 & 1.82 & 10.79 & 1.88 \\
1.00 & 0.05 & 3.26 & 1.96 & 9.70 & 2.01 \\
2.00 & 0.18 & 6.55 & 0.67 & 10.40 & 0.85 \\
3.00 & 0.25 & 7.60 & 0.28 & 8.65 & 0.53 \\
4.00 & 0.28 & 7.21 & 0.30 & 8.41 & 0.58 \\
5.00 & 0.31 & 7.31 & 0.25 & 7.75 & 0.56 \\
\hline
\end{tabular}
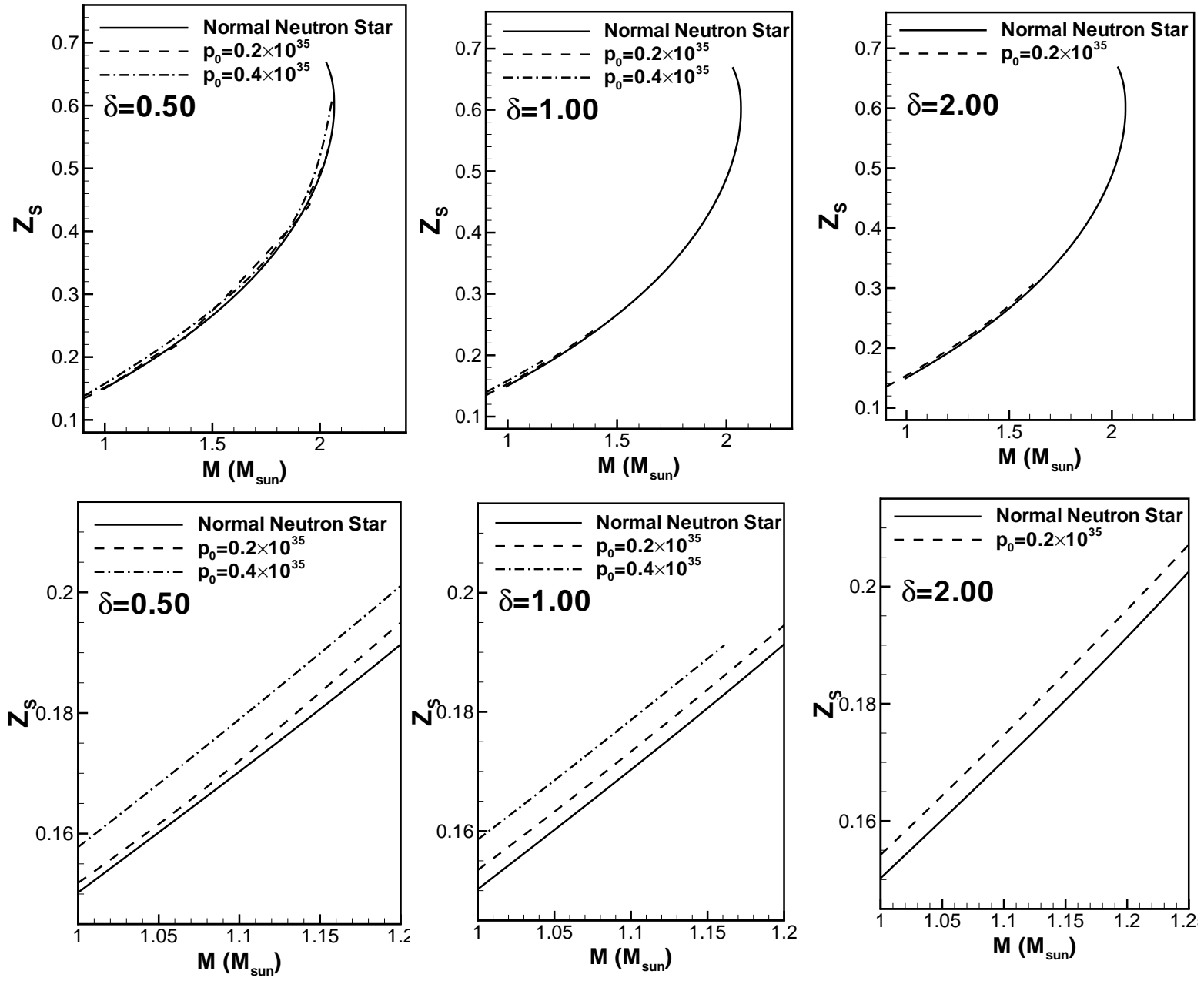

Figure 15. Top: gravitational redshift, $Z_{s}$, versus total mass of DMANSs with different DMEOSs from the velocity profile of galaxies and central pressure ratio, $\delta$. Bottom: same as the top, but for a different range of DMANS mass.

However, the visible radius of the DMANS is smaller than the normal neutron star. Therefore, the gravitational redshift of spectral lines can be used to investigate these two stars. Figures 15-18 present the gravitational redshifts of a normal neutron star and DMANSs with different DMEOSs, and various values of central pressure ratio. In all cases, the existence of DM leads to higher values of gravitational redshift compared with normal neutron stars, in agreement with previous reports (Leung \& Chu \& Lin 2011; Xiang et al. 2014). Moreover, the gravitational redshift in the DMANSs with $m=1.0 \mathrm{GeV}$ is larger than in the cases with $p_{0}=0.2$ and $p_{0}=0.4 \times 10^{35} \mathrm{dyn}_{\mathrm{cm}}^{-2}$. Figures 15-18 indicate that the effects of DM on the gravitational redshift are more significant in stars with the higher values of $\delta$. These results can be used to distinguish the normal neutron stars from DMANSs with different DMEOSs and contributions of DM. 

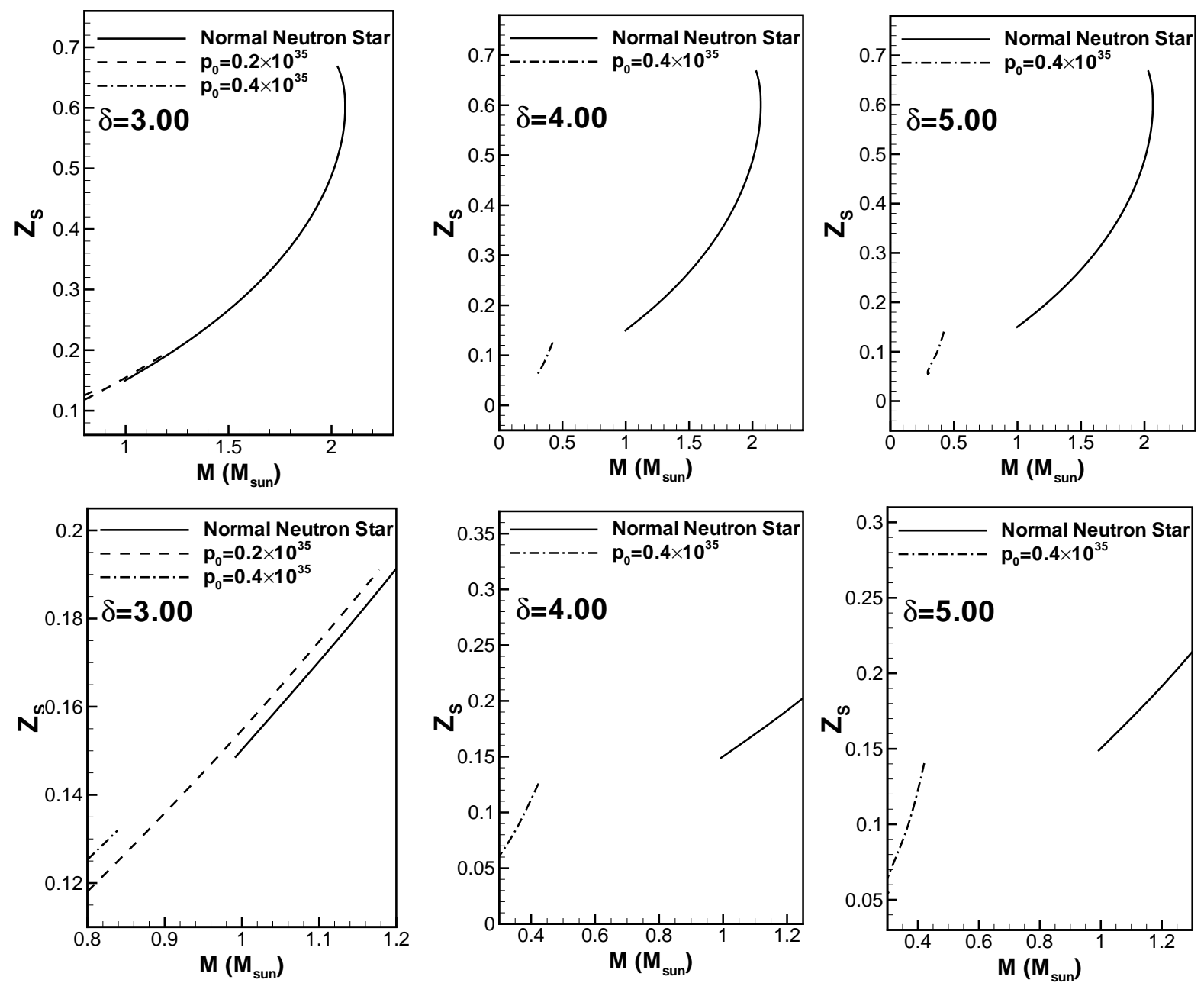

Figure 16. Same as Figure 15 but for other values of central pressure ratio, $\delta$.

\section{SUMMARY AND CONCLUSIONS}

Applying the DMEOSs from the rotational curves of galaxies, as well as the fermionic DMEOS with $m=1.0 \mathrm{GeV}$ and the NM EOS from Skyrme effective force, we have studied the structure of DMANSs. We have found that the radius of a DMANS is smaller than the radius of a normal neutron star with the same mass. Our results show that the mass-radius relations are affected by both the DMEOS and central pressure ratio of DM to NM. Specifically, depending on the DMEOS and the central pressure ratio, the DMANS can be a gravitationally or self-bound star. Additionally, it has been confirmed that in some cases two DMANSs with equal visible radii but different masses can exist. This fact is a consequence of different degrees of DM domination in DMANSs. We have shown that, with all DMEOSs, the maximum mass of the DMANS is lower than the normal neutron star. The observed neutron stars EXO 1745-248, 4U 1608-52, and 4U 1820-30, which are inconsistent with the previous observations and theoretical properties of normal neutron stars, can be explained with our model. We have confirmed that some DMEOSs and central pressure ratios considered in this work result in DM-dominated neutron stars with a halo of DM around a small NM sphere. Our calculations suggest that the observed neutron stars EXO 1745-248, 4U 1608-52, and 4U 1820-30 may be DM-dominated neutron stars. It has been clarified that the radius of the NM sphere decreases while the radius of the DM sphere increases as the central pressure ratio grows. In addition, the mass of the DM sphere increases with the increase of central pressure ratio. Moreover, the DMANSs with high values of central pressure ratio are DM-dominated neutron stars. Finally, we have shown that the existence of DM in the DMANSs results in increased gravitational redshift. 

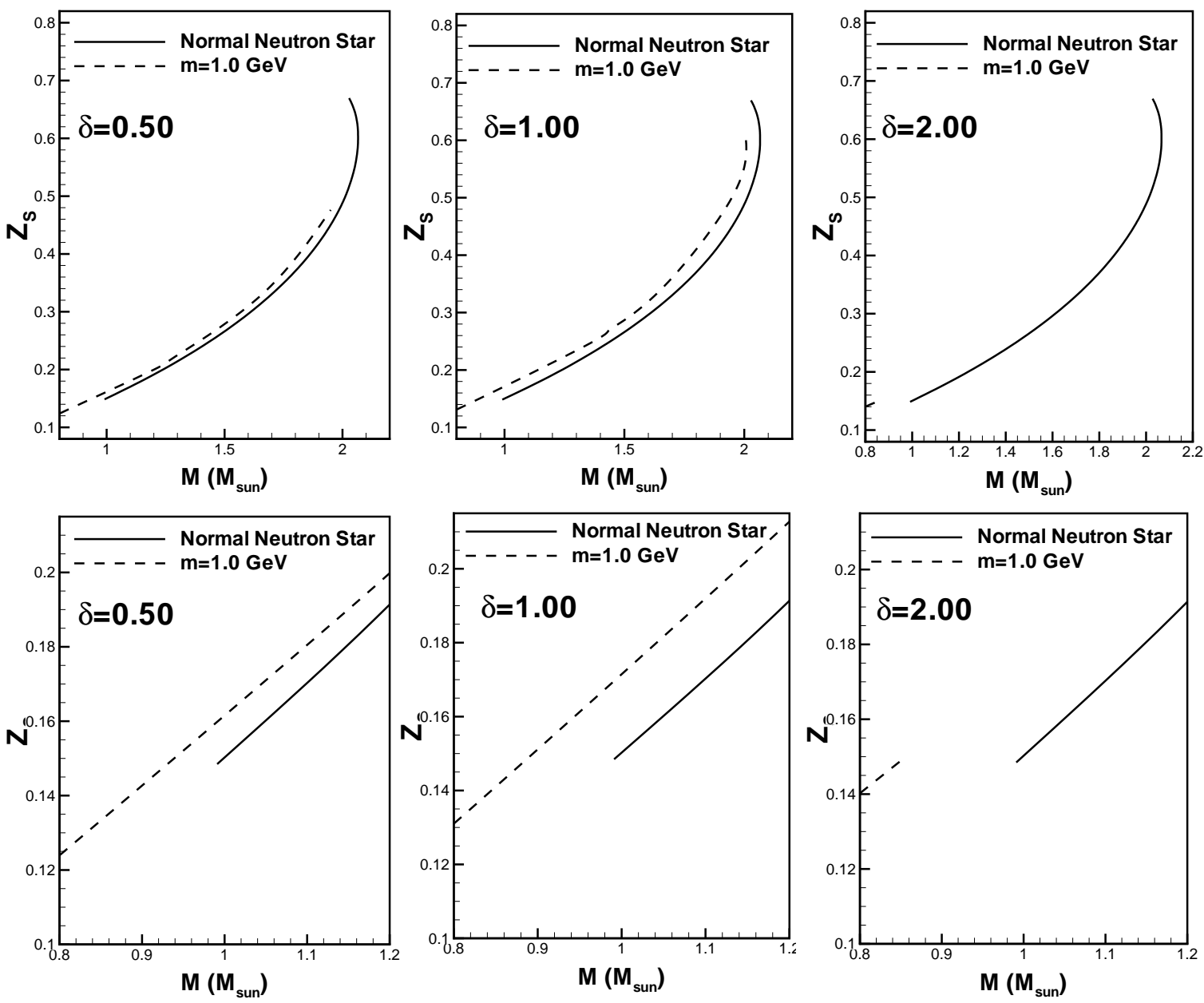

Figure 17. Same as Figure 15 but for DMANSs with the fermionic DMEOS for different central pressure ratios, $\delta$.

The author wishes to thank the Shiraz University Research Council and the Iran Science Elites Federation.

\section{REFERENCES}

Ade, P. A. R., Aghanim, N., Armitage-Caplan, C., et al. 2014, ArXiv e-prints: 1303.5076v3

Akmal, A., \& Pandharipande, V.R. 1997, PhRvC, 56, 2261

Alford, M., Blaschke, D., Drago, A., et al. 2007, Natur, 445, E7

Antoniadis, J., Freire, P.C.C., Wex, N., et al. 2013, Sci, 340, 448

Barranco J., Bernal A., \& Nunez D. 2015, MNRAS, 449, 403

Baym G., Pethick C., \& Sutherland P. 1971, ApJ, 170, 299

Bertone G., \& Fairbairn M. 2008, PhRvD, 77, 043515

Chabanat, E., Bonche, P., Haensel, P., Meyer, J., \& Schaeffer, R. 1997, NuPhA, 627, 710

Ciarcelluti P., \& Sandin F. 2011, PhLB, 695, 19

Demorest, P. B., Pennucci, T., Ransom, S. M., Roberts, M. S. E., \& Hessels, J. W. T. 2010, Natur, 467, 1081

Engvik, L., Hjorth-Jensen, M., Osnes, E., Bao, G., \& ?stgaard, E. 1994, PhRvL., 73, 2650

Fuller J., \& Ott, C. D. 2015, MNRAS, 450, L71

Goldman I., \& Nussinov S. 1989, PhRvD, 40, 3221

Guver, T., Ozel, F., Cabrera-Lavers, A., \& Wroblewski, P. 2010, ApJ, 712, 964

Guver, T., Wroblewski, P., Camarota, L., \& Ozel, F. 2010, ApJ, 719, 1807

Klahn, T., Blaschke, D., Sandin, F., et al. 2007, PhLB, 654, 170
Kouvaris C. 2008, PhRvD, 77, 023006

Kouvaris C. 2012, PhRvL, 108, 191301

Lattimer J. M., \& Prakash M. 2001, ApJ, 550, 426

Lattimer J. M. \& Prakash M. 2007, PhR, 442, 109

de Lavallaz, A., \& Fairbairn M. 2010, PhRvD, 81, 123521

Leung S.-C., Chu M.-C., \& Lin L.-M. 2011, PhRvD, 84, 107301

Leung S.-C., Chu M.-C., \& Lin L.-M. 2012, PhRvD, 85, 103528

Li A., Huang F., \& Xu R.-X. 2012, APh, 37, 70

Li, A., Zhou, X. R., Burgio, G. F., \& Schulze, H. -J. 2010, $\mathrm{PhRvC}, 81,025806$

Massey, R., Rhodes, J., Ellis, R., et al. 2007, Natur, 445, 286

Narain, G., Schaffner-Bielich, J., \& Mishustin, I. N. 2006,

PhRvD, 74, 063003

Ozel F. 2006, Natur, 441, 1115

Ozel F., Guver T., \& Psaltis D. 2009, ApJ, 693, 1775

Page D.\& Reddy S. 2006, ARNPS, 56, 327

Perez-Garcia M. A., \& Silk J. 2012, PhLB, 711, 6

Perez-Garcia M. A., Silk J., \& Stone J. R. 2010, PhRvL, 105, 141101

Prakash, M., Ainsworth, T.L. \& Lattimer, J.M. 1988, PhRvL, 61,2518

Sandin F., \& Ciarcelluti P. 2009, APh, 32, 278 

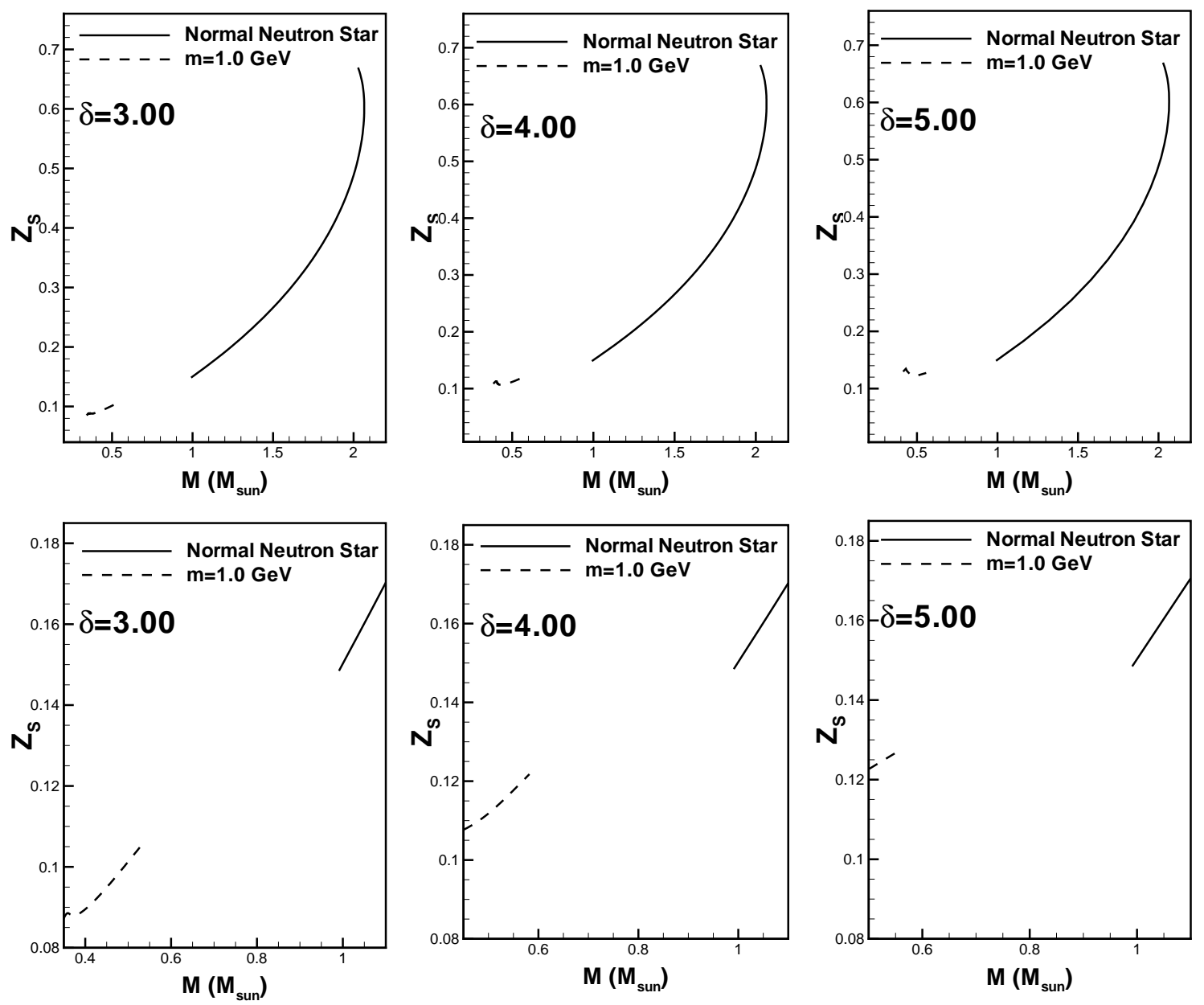

Figure 18. Same as Figure 17 but for other values of central pressure ratio, $\delta$.

Serra A. L., \& Dominguez Romero M. J. L. 2011, MNRAS, 415, L74

Springel, V., White, S. D. M., Jenkins, A., et al. 2005, Natur, 435,629
Weber F. 2005, PrPNP, 54, 193

Weber M., \& de Boer W. 2010, A \& A, 509, A25

Xiang, Q.-F., Jiang, W.-Z., Zhang, D.-R., \& Yang, R.-Y. 2014, $\mathrm{PhRvC}, 89,025803$

\section{ALL AUTHORS AND AFFILIATIONS}

AND

Z. REZAEI.

Physics Department and Biruni Observatory

College of Sciences, Shiraz University

Shiraz 71454, Iran 